\section{Bugs and the gut: breaking barriers to understanding}

There has been a re-emergence of interest in the interplay between the faecal microbiota and its associated metabolisms and gastrointestinal function. This has recently been driven by the current interest in human and veterinary probiotics, and in the recent past by hypotheses which linked gut bacterial metabolism of ingested foodstuffs and colorectal and other cancers. However, what is less widely known is the extent of involvement of gastrointestinal bacteria in gut function, gut disease, and particularly diseases of other organs.

Some of the less generally well known aspects of this interplay relate to gut bacterial metabolites: for example, the production of volatile fatty acids exacerbating acidaemias such as methylmalonic acidaemia, and the associated role of antibiotics in patient management. ${ }^{1}$ An area where there has been a resurgence of interest is the production of phytooestrogens from dietary precursors and their possible role in protection from hormone dependent breast cancer. $^{23}$ The role of such metabolites in infertility in humans remains unexplored. There is even evidence that gut bacteria can induce or repress liver cytochrome P450 due to metabolism of dietary glucosinolates. ${ }^{4}$

There is a great deal of evidence to support the fact that what is considered normal gut structure and function is the end point of a complex set of interactions between the host and microbes colonising the gut. ${ }^{5}$ Such normal features include gastrointestinal motility, secretion and absorption, cell composition, mitotic activity, and villous length, etc. To this we can now add the fact that normal components of the gut microbiota can alter colonic barrier function by modifying colonic wall permeability (see page 503$)^{6}$. This interesting observation raises a whole series of questions. The rationale behind the study is the hypothesis that increased intestinal permeability is a key factor in the pathogenesis of idiopathic bowel disease and the proposal that permeability may be affected by gut bacteria. What was found in the rat model used was that none of the test organisms increased permeability to a large molecular probe (dextran) but some altered permeability to a small molecular probe (mannitol). Interestingly, this modification in permeability was not uniform, with reduction, increase, and no significant alteration being detected, dependent on the test organism used. What would be interesting to know is the extent to which such effects are a feature of the bacterial strain used or a feature of the bacterial species represented. For example, do most commensal Escherichia coli increase permeability or only a few strains? This leads to the deeper question of whether or not patients with idiopathic bowel inflammation are more frequently colonised with bacteria that increase gut permeability and/or have a gut microbiota where the net effect is to increase permeability. Both questions can now be answered. I would be particularly interested to see the effect of crude gut "flora" from, for example, patients with Crohn's disease, on gut wall permeability in small and large bowel in the "rat-human flora" model.

Similarly, it would be of interest to know whether or not the decrease in permeability induced by the isolate of Lactobacillus brevis is strain specific or a general feature in the species or genus. This may be of particular relevance in the probiotic field. A minor disappointment with the current paper is that the opportunity was not taken to see if coadministration of the $L$ brevis strain with the $E$ coli strain would reverse or prevent the increased permeability associated with $E$ coli.

There are many other questions which come to mind such as: could an ability to increase permeability be a virulence factor which facilitates translocation of a bacterium or its toxin - for example, verotoxin across the gut wall? Are changes in permeability due to specific factors produced by these bacteria or are they a result of metabolic byproducts? What is the range of degrees of altered permeability induced by different bacteria?

I have little doubt that this paper will stimulate research in these areas and that the results of such studies will offer increasing insights into the complex interplay between the host and its microbiota, and a better understanding of what is normal and what is abnormal.

P BORRIELLO

PHLS Central Public Health Laboratory,

61 Colindale Avenue, London NW9 5HT, UK

pboriello@phls.nhs.uk 1 Bain MD, Jones M, Borriello SP, et al. Contribution of gut bacterial
metabolism to human metabolic disease. Lancet 1988;1:1078-9.
2 Setchell KDR, Borriello SP, Hulme P, et al. Non-steroidal oestrogens of
dietary origin: Possible roles in hormone dependent disease. Am f Clin Nutr
$1984 ; 40: 569-78$.
3 Ingram D, Sanders K, Kolybaba $\mathrm{M}$, et al. Case-control study of
phyto-oestrogens and breast cancer. Lancet $1997 ; 350: 990-4$.
4 Nugon-Baudon L, Robot S, Flinois JP, et al. Effects of the bacterial status of
rats on the changes in some liver cytochrome P450 (EC 1.14.14.1)
apoproptein consequent to a glucosinolate-rich diet. Br $\mathcal{f}$ Nutr 1998;80:
$231-4$.
5 Borriello SP. Bacteria and gastrointestinal secretion and motility. Scand $\mathcal{f}$
Gastroenterol $1984 ; 19$ (suppl 93 ):115-21.
6 García-Lafuente A, Antolín M, Guarner F, et al. Modulation of colonic bar-
rier function by the composition of the commensal flora in the rat. Gut
2001;48:503-7.

\section{The changing scope of colorectal cancer}

The prognosis in colorectal cancer depends on the stage at which the disease is diagnosed. Patients with advanced disease usually die of cancer, but when large intestinal tumours are found at an early asymptomatic phase a cure can be anticipated. Furthermore, the premalignant lesions - adenomatous polyps-grow in the colon for years and perhaps decades before malignant conversion occurs, providing an opportunity for their removal, interrupting the natural history of these neoplasms. It has long been recognised that preventive strategies would be appropriate for this disease, and an extensive literature can be found on the subject

Two general approaches to detecting asymptomatic early staged colorectal cancers have been studied in dept. The first is the use of faecal occult blood tests as colorectal neoplasms 
add blood to the stool that can be detected prior to the development of symptoms. However, this approach is relatively weak due to deficiencies in both sensitivity and specificity. One can expect a reduction of colorectal cancer mortality of $<20 \%$ if the test is performed every other year. ${ }^{1-3}$ False positive tests greatly outnumber true positives, all of which require a full investigation. The test is easy and noninvasive, but compliance is poor. This approach is often used but enthusiasm for this should be limited. The second approach is to perform endoscopic screening on asymptomatic individuals and remove the premalignant lesions. Although all of the evidence for efficacy of the latter approach is either retrospective or uncontrolled, the reduction in cancer mortality might be as high as $70-80 \%$, and it may be sufficient to perform these examinations only once every $5-10$ years to achieve this outcome. ${ }^{45}$

Flexible sigmoidoscopy will readily detect neoplasms as far as the splenic flexure in unsedated patients. The traditional autopsy based literature from the mid-20th century indicated that as many as $75 \%$ of all colorectal neoplasms occurred in the distal colon. With this distribution, sigmoidoscopy would be an excellent (though imperfect) means of finding early colorectal cancers. However, if the distribution of colorectal lesions should shift, the impact of this procedure would change accordingly. It has been reported from North America and Europe that over the past several decades the location of colorectal neoplasms is shifting from the left to the right side of the colon. McCallion et al have communicated in this issue of Gut that when the distribution of colorectal cancers in Northern Ireland reported from 1990-97 was compared with those reported from 1976-78, a significant proximal shift had occurred; whereas only $23.5 \%$ of cancers were found proximal to the splenic flexure in the early time period, this number grew to $36.7 \%$ in the 1990 s (see page 522). ${ }^{6}$ Is this really the case, and if so, what accounts for it?

Firstly, did this apparent proximal shift really occur or was it an artefact of data collection? It is possible that previously under counted proximal cancers are more accurately diagnosed by the use of current technology, including colonoscopy and imaging techniques. One would need to know how often patients died without an accurate diagnosis of a proximal colon cancer in the 1970s, and this may be difficult or impossible to solve. It is probably the case that there has been a fall in the number of deaths in the Northern Ireland registries that were attributed to abdominal cancer of unknown source, and the assumption is that proximal rather than distal cancers would have been more likely undiagnosed. It is perhaps worth noting that the two databases used by McCallion et al had substantial differences in ascertainment: the Northern Ireland Cancer Registry (NICR) reported 922.3 colorectal cancers/year whereas the Northern Ireland CRC registry reported only 644.1 year, as pointed out by the authors. The authors have rejected a significant impact of these differences, but in the NICR database, nearly $20 \%$ of colorectal cancers were of an unknown site.

Secondly, if this pattern shift is true, what is the mechanism for this change? A growth in public awareness of symptoms and prompt diagnosis of symptomatic cancers may lead to an improvement in mortality, but not in cancer incidence, which would remain unchanged. If a substantial proportion of the population had been subjected to screening sigmoidoscopy with the attendant removal of adenomas, this might lead to a substantial reduction in cancer incidence, especially in the distal colon and rectum. The authors do not present data on the frequency of flexible sigmoidoscopy for colon cancer screening during the two periods but it is possible that there had been an increased use of flexible sigmoidoscopy (for screening or symptoms) during the 1990-97 period compared with the $1976-78$ period. Such has been the case in the USA. If the later study group consisted of a more elderly population, it is expected that there would be a greater proportion of right sided colon cancers. ${ }^{7}$ Finally, perhaps another factor has intervened to alter the distribution of cancers within the colon. It has been demonstrated that aspirin and non-steroidal anti-inflammatory users experience a reduction in cancer mortality in the range of $40 \%$, even for relatively occasional users. ${ }^{8}{ }^{9}$ If additional factors such as increased caloric intake raised the general risk for cancer in the population while aspirin and related agents reduced the risk in the distal colon, there would appear to be a left to right shift at the population level. This remains a conjecture at present but it is likely that the factors responsible for the pathogenesis of proximal cancers may be quite different from those that lead to distal cancers.

Finally, what is the clinical impact of a true shift in colorectal neoplasms towards the proximal colon? Recent publications from North America explore the impact of screening with total colonoscopy. ${ }^{10}{ }^{11} \mathrm{~A}$ substantial number of patients have important lesions above the reach of the sigmoidoscope without distal "signal" lesions. Patients in the USA have access to this information through the news media and Internet, and increasingly are insisting on a complete colonoscopic examination, particularly if they perceive themselves to be at an increased risk for cancer. Considerable investments of resources are at stake in this debate. Perhaps we should accept that only a full examination of the colon will suffice for purposes of screening. This would add pressure to more fully develop highly accurate non-invasive imaging techniques to screen the entire colon, such as virtual colonoscopy. Eventually genetic testing of stools might be sufficiently sensitive to act as a primary screening test. ${ }^{12}$ We have come a long way since the first stool was tested with guaiac reagents. We need to adapt to the changing nature of the disease that might be occurring as we design optimal prevention programmes.

\section{R BOLAND}

T J SAVIDES

Department of Medicine and Cancer Center

University of California, San Diego, San Diego, California, USA

Correspondence to: Dr C R Boland, 9500 Gilman Drive, San Diego, CA 92093-0688, USA. crboland@ucsd.edu

1 Hardcastle JD, Chamberlain JO, Robinson $\mathrm{MH}$, et al. Randomised controlled trial of faecal-occult-blood screening for colorectal cancer. Lancet 1996;348:1472-7.

2 Kronborg O, Fenger C, Olsen J, et al. Randomised study of screening for colorectal cancer with faecal-occult-blood test. Lancet 1996;348:1467-71. 3 Mandel JS, Church TR, Bond JH, et al. The effect of fecal occult-blood screening on the incidence of colorectal cancer. $N$ Engl $f$ Med 2000;343:1603-7.

4 Selby JV, Friedman GD, Quesenberry CP jr, et al. A case-control study of screening sigmoidoscopy and mortality from colorectal cancer. $N$ Engl $\mathcal{F}$ Med 1992;326:653-7.

5 Winawer SJ, Zauber AG, O'Brien MJ, et al. Randomized comparison of surveillance intervals after colonoscopic removal of newly diagnosed adenomatous polyps. The National Polyp Study Workgroup. N Engl f Med 1993;328:901-6

6 McCallion K, Mitchell RMS, Wilson RH, et al. Flexible sigmoidoscopy and the changing distribution of colorectal cancer: implications for screening. Gut 2001;48:522-5.

7 Saltzstein SL, Behling CA, Savides TJ. The relation of age, race, and gender to the subsite location of colorectal carcinoma. Cancer 1998;82:1408-10.

8 Thun MJ, Namboodiri MM, Heath CW jr. Aspirin use and reduced risk of fatal colon cancer. N Engl f Med 1991;325:1593-6.

9 Giovannucci E, Egan KM, Hunter DJ, et al. Aspirin and the risk of colorectal cancer in women. $N$ Engl f Med 1995;333:609-14.

10 Lieberman DA, Weiss DG, Bond JH, et al. Use of colonoscopy to screen asymptomatic adults for colorectal cancer. Veterans Affairs Cooperative Study Group 380. N Engl f Med 2000;343:162-8.

11 Imperiale TF, Wagner DR, Lin CY, et al. Risk of advanced proximal neoplasms in asymptomatic adults according to the distal colorectal findings. $N$ Engl f Med 2000;343:169-74.

12 Ahlquist DA, Skoletsky JE, Boynton KA, et al. Colorectal cancer screening by detection of altered human DNA in stool: feasibility of a multitarget assay panel. Gastroenterology 2000;119:1219-27. 